\title{
BMJ Open Impact of Compression Therapy on Cellulitis (ICTOC) in adults with chronic oedema: a randomised controlled trial protocol
}

\author{
Elizabeth Webb, ${ }^{1,2}$ Teresa Neeman, ${ }^{3}$ Jamie Gaida, ${ }^{4}$ Francis J Bowden, ${ }^{5,6}$ \\ Virginia Mumford, ${ }^{\oplus 7}$ Bernie Bissett ${ }^{2}$
}

To cite: Webb E, Neeman T, Gaida J, et al. Impact of Compression Therapy on Cellulitis (ICTOC) in adults with chronic oedema: a randomised controlled trial protocol. BMJ Open 2019;9:e029225. doi:10.1136/ bmjopen-2019-029225

- Prepublication history for this paper is available online. To view these files, please visit the journal online (http://dx.doi. org/10.1136/bmjopen-2019029225).

Received 17 January 2019 Revised 30 June 2019 Accepted 3 July 2019

\section{Check for updates}

(c) Author(s) (or their employer(s)) 2019. Re-use permitted under CC BY-NC. No commercial re-use. See rights and permissions. Published by BMJ.

For numbered affiliations see end of article.

\section{Correspondence to}

Elizabeth Webb;

elizabeth.webb@calvary-act. com.au

\section{ABSTRACT}

Introduction Cellulitis represents a significant burden to patients' quality of life (QOL) and cost to the healthcare system, especially due to its recurrent nature. Chronic oedema is a strong risk factor for both an initial episode of cellulitis and cellulitis recurrence. Expert consensus advises compression therapy to prevent cellulitis recurrence in individuals with chronic oedema, however, there is little supporting evidence. This research aims to determine if the management of chronic oedema using compression therapy effectively delays the recurrence of lower limb cellulitis.

Methods and analysis A randomised controlled trial with cross-over will be used to assess the impact of compression therapy on clinical outcomes (time to next episode of cellulitis, rate of cellulitis-related hospital presentations, QOL and leg volume). Using concealed allocation, 162 participants will be randomised into the intervention (compression) or control (no compression) group. Randomisation will be stratified by prophylactic antibiotic use. Participants will be followed up at 6 monthly intervals for up to 3 years or until 45 episodes of cellulitis occur across the cohort. Following an episode of recurrent cellulitis, control group participants will cross-over to the intervention group. Survival analysis will be undertaken to assess the primary outcome measure of time to cellulitis recurrence. The hypotheses are that compression therapy to control lower limb chronic oedema will delay recurrent lower limb cellulitis, reduce the rate of associated hospitalisations, minimise affected limb volume and improve the QOL of this population.

Ethics and dissemination Ethics approval has been obtained from the ethics committees of all relevant institutions. Results will be disseminated through relevant peer-reviewed journal articles and conference presentations.

Trial registration number ACTRN12617000412336; Preresults. The ICTOC trial is currently in progress. Participant recruitment started in May 2017 and is expected to continue until December 2019.

\section{BACKGROUND AND RATIONALE}

Cellulitis is a common acute bacterial infection of the skin and subcutaneous tissue. ${ }^{1}$ The majority of cellulitis episodes $(69 \%-81 \%)$
Strengths and limitations of this study

- Randomisation of participants will be stratified by prophylactic antibiotic use to ensure antibiotic use does not confound treatment outcome.

- Due to the nature of the intervention, blinding is not feasible for participants or assessors.

- Assessment tools and methods (perometer, diagnosis of cellulitis by medical practitioners external to the trial, verification of data using the medical record or general practitioner) have been selected to minimise potential measurement bias.

- The use of broad inclusion criteria will allow for trial results to be generalised to adults across a range of settings nationally and internationally.

occur in the lower limbs.. ${ }^{2-4}$ In Australia, lower limb cellulitis is associated with significant health costs due to frequent hospital admissions and high levels of morbidity. In 2014-2015, there were 59466 hospitalisations for cellulitis, ${ }^{5}$ with the average admission lasting 4.3 days. $^{6}$ In 2013-2014, cellulitis was the third leading cause of potentially preventable hospital admissions, with over half of all admissions for cellulitis being considered potentially preventable. ${ }^{6} 7$ Erysipelas is an infection similar to cellulitis, which typically affects more superficial tissues. As the terms erysipelas and cellulitis are often used interchangeably and most clinical studies do not differentiate between them, this paper will consider them as one entity.

Recurrence of cellulitis is common and represents a significant proportion of the disease burden. In a 3-year time frame cellulitis has been reported to recur in $29 \%-47 \%$ of patients, ${ }^{89}$ with a case series in Sweden finding that $13 \%$ of patients admitted for cellulitis developed two or more recurrences within 3 years. ${ }^{9}$ In light of the significant recurrence rates, effective interventions 
which reduce recurrence could limit the disease burden and improve patient outcomes.

Oedema occurs when capillary filtration overwhelms the available lymphatic drainage. ${ }^{10}$ Lymphoedema specifically refers to persistent oedema resulting from lymphatic drainage failure. ${ }^{11}$ Chronic oedema is an umbrella term that refers to oedema resulting from insufficient lymphatic drainage, where the principal cause of oedema may be increased capillary filtration and/or lymphatic drainage failure. ${ }^{11}$ As such, the term chronic oedema encompasses oedema of various aetiologies, including lymphoedema. For the purpose of this trial, we will use the term chronic oedema.

Lymphoedema and chronic oedema are potent risk factors for developing lower limb cellulitis and for its recurrence. ${ }^{481213}$ It is broadly accepted that the relationship between cellulitis and chronic oedema is a vicious cycle. ${ }^{8}{ }^{14}$ Chronic oedema predisposes individuals to cellulitis and with each episode of cellulitis, the lymphatic system is further impaired, increasing residual oedema and heightening risk of future cellulitis infections. ${ }^{14}$ Thus, chronic oedema is not only a result of cellulitis but also increases the risk of recurrence. ${ }^{14}$

The standard treatment for chronic oedema includes compression therapy and skin care. ${ }^{15}$ Compression bandaging can be used to reduce oedema in a limb, and daily wear of compression garments is used to control oedema. There is general consensus that in addition to antibiotic prescription, compression to manage oedema should be an adjuvant treatment for patients with chronic oedema who are experiencing cellulitis recurrence. ${ }^{181416}$ Despite this common recommendation and the strong evidence supporting the relationship between oedema and cellulitis, there is a paucity of evidence to support the use of compression to manage chronic oedema to prevent cellulitis recurrence.

The time-intensive nature of compression therapy and the fact that measuring meaningful outcomes requires lengthy assessment periods probably contribute to the lack of research in this field. Only one study has been conducted on the impact of oedema management on cellulitis recurrence, ${ }^{17}$ with a second study incidentally observing a reduction in 'infection' among patients receiving oedema management, although this was not a research objective. ${ }^{18}$ While both studies support the hypothesis that oedema management decreases cellulitis recurrence, their conclusions are hampered by methodological limitations, including pre-post intervention methods, small sample sizes and change in infection rate not being specified a research objective. ${ }^{17}{ }^{18}$ While research regarding compression therapy to prevent cellulitis recurrence is scarce, there is high-quality evidence to support the use of prophylactic antibiotics. A multicentre, double-blind, randomised controlled trial (RCT) found that the use of prophylactic antibiotics in patients experiencing recurrent cellulitis is effective in preventing subsequent attacks, although the effect diminishes following prophylaxis cessation. ${ }^{19}$ A 2017 Cochrane systematic review of interventions to prevent cellulitis identified six studies investigating prophylactic antibiotics, but no other randomised trials investigating other prophylactic measures such as oedema management or skin care. ${ }^{20}$ Thus further research into the efficacy of prophylactic measures other than antibiotic is warranted..$^{20}$

The following protocol describes a RCT with cross-over to determine if the use of compression therapy for adults experiencing lower limb recurrent cellulitis and chronic oedema will delay cellulitis recurrence.

\section{RESEARCH HYPOTHESES}

The hypotheses are that compression therapy to control lower limb chronic oedema will delay recurrent lower limb cellulitis, reduce the rate of associated hospitalisations, minimise affected limb volume and improve the quality of life (QOL) of this population.

\section{RESEARCH OBJECTIVES Primary objective}

The primary objective was to determine if compression therapy delays the recurrence of lower limb cellulitis in adults with lower limb chronic oedema and recurrent cellulitis.

\section{Secondary objectives}

The secondary objectives were to determine if, in adults with lower limb chronic oedema and recurrent cellulitis, compression therapy: (1) reduces the rate of cellulitis-related hospital presentations; (2) reduces affected leg volume; and (3) improves QOL.

\section{TRIAL DESIGN}

A RCT with cross-over will be used to assess the impact of compression therapy on clinical outcomes (time to next episode of cellulitis, rate of cellulitis-related hospital presentations, QOL and leg volume). Participants will be randomised to the intervention or control group by block randomisation using sealed opaque envelopes. As prophylactic antibiotics have been shown to influence cellulitis recurrence, ${ }^{19-21}$ randomisation of participants will be stratified by prophylactic antibiotic use. Following an episode of cellulitis, participants in the control group will crossover into the intervention group, whereas intervention group participants will remain in their original group and continue to receive compression therapy. Figure 1 shows the proposed participant allocation process.

The absence of high-quality evidence regarding the impact of compression therapy on recurrence of cellulitis means there is uncertainty as to whether it is an effective intervention, justifying the use of an RCT. Although there is no high-quality evidence to support the use of the compression therapy to prevent cellulitis in this patient population, it reflects the accepted expert opinion and the standard clinical practice of the institution conducting the trial. 


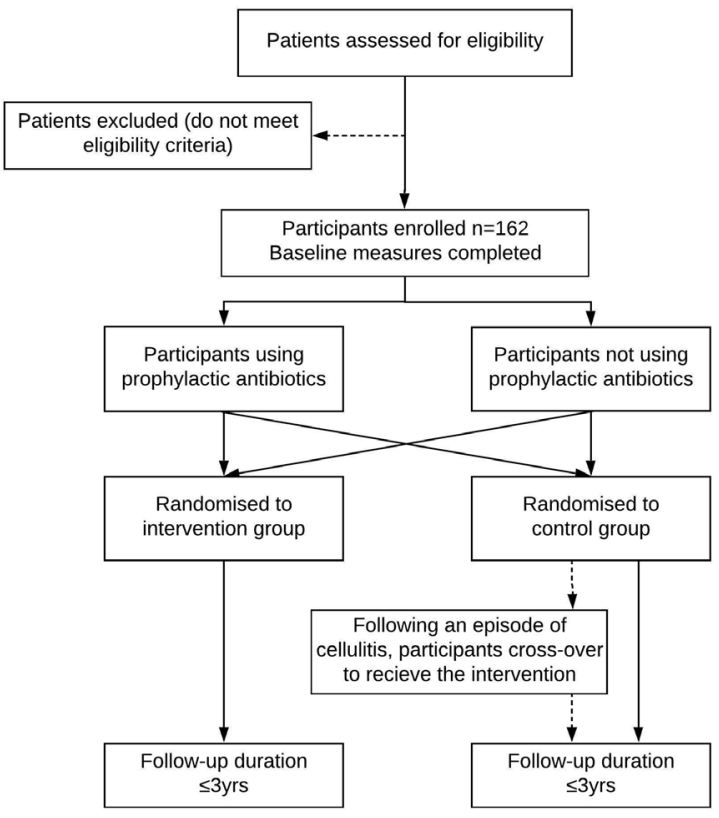

Figure 1 Anticipated participant flow through trial.

Therefore, the trial design crosses the control group participants over into the intervention group following the first episode of cellulitis to ensure no participant continues to experience recurrent cellulitis episodes without receiving the institution's standard intervention.

\section{METHODS}

\section{Study setting and population}

The trial will be conducted at the Calvary Public Hospital Bruce (CPHB) outpatient lymphoedema clinic. Adults with lower limb chronic oedema and a history of recurrent cellulitis who meet the eligibility criteria will be recruited from the two major ACT public hospitals (CPHB and Canberra Hospital) and general practitioners servicing the ACT and nearby NSW residents.

\section{Eligibility criteria}

Inclusion criteria

$\checkmark \geq 18$ years of age.

- Two or more episodes of cellulitis diagnosed in the same leg in the past 2 years (at the time of referral). Clinical diagnosis of cellulitis ideally will have been based on the presence of acute erythema, oedema, warmth and pain, with spreading involvement of the skin and subcutaneous tissues, malaise and possibly fever. $^{12223}$

- Chronic oedema (oedema persisting $\geq 3$ months) in the $\operatorname{leg}(\mathrm{s})$ that have had recurrent cellulitis diagnosed (presence of oedema confirmed by an accredited lymphoedema therapist through interview and physical examination, including a thorough medical history combined with limb palpation and visual assessment).
- Understanding of involvement in the study as per the participant information sheet.

- Provision of informed consent.

- Able to attend regularly scheduled appointments for the duration of the study.

- Has a valid Medicare number.

\section{Exclusion criteria}

- Currently wearing effective compression garments ( $\geq$ compression class 2, or compression class 1 if considered effective by a lymphoedema therapist) regularly ( $\geq 5$ days per week).

- Declines to participate or is unable to participate for whatever reason.

- Receiving end of life care.

- Medically unstable.

- Chronic wound/ulcer, or a wound/ulcer requiring specialist treatment or treatment that prevents the use of compression garments.

- Unable to wear compression (unable to don/doff garments or has a medical condition that contraindicates the use of compression).

\section{Interventions}

All assessments, interventions and outcome measures will be conducted by a physiotherapist or occupational therapist who meets the registration requirements for category one of the Australian National Lymphoedema Practitioners Register. ${ }^{24}$

At the initial appointment, eligibility will be formally checked, and those who consent to participate will undergo stratified randomisation using sealed, opaque and identical envelopes that are sequentially numbered. Prior to randomisation, baseline measures including number of episodes of cellulitis in the 2 years prior to referral, duration of chronic oedema, referral source and demographics will be captured. The presence of identified potential risk factors for cellulitis will also be recorded, including history of tinea or other fungal infections between toes, diabetes mellitus, obesity and chronic venous insufficiency. ${ }^{3412} 2526$

At the initial appointment, participants in both the control and intervention groups will receive education (verbal and written) regarding cellulitis and how to decrease the risk of recurrence. Education will include the benefits of skin care, prevention of tinea or other fungal infections between toes, maintaining healthy body weight and regular exercise.

For the intervention group, the initial appointment will also be used to plan appropriate compression therapy which will be provided at subsequent appointments. Compression therapy will involve the application of compression garments (compression stockings or wraps) and may or may not involve compression bandaging to minimise oedema prior to the fitting of compression garments. The number of appointments necessary for the provision of compression therapy will be individualised to meet participant requirements. 
Participants in both groups will be followed for up to 3 years at 6 monthly intervals (table 1 ) to complete outcome measures and to continue to receive the allocated treatment (education with or without compression therapy). At each appointment, the therapist will inform each participant of changes in their limb volume, providing tangible feedback to support ongoing participant attendance. Throughout the trial, participants in the intervention group may require additional appointments for compression therapy (compression bandaging, and measure for and provision of compression garments). Intervention compliance (number of days per week garments are worn) and adverse effects will be captured by self-report.

Cross-over of control group participants will be triggered on clinician identification of cellulitis. The recurrence of cellulitis will be checked at scheduled appointments, however, if a participant reports a recurrence between scheduled assessments, they will be reviewed at an additional appointment to record outcome measures (table 1), and to commence cross-over for control group participants. Date of cross-over will be defined as the day compression garments are initially fitted.

\section{Outcome measures}

Table 1 shows the timeline for completion of trial activities and outcome measures.

The primary outcome is 'observed time to the first episode of cellulitis recurrence'. Cellulitis recurrence will only be assessed in a leg that has been assessed as having chronic oedema, thus if cellulitis occurs in a leg that was not previously identified as having chronic oedema, the infection will not be considered a recurrence. Cellulitis will be diagnosed by medical practitioners external to the study. Date of cellulitis recurrence (and associated hospitalisation) will be gained by participant self-report and may be verified using medical records from the hospitals and/or general practitioners.

Secondary outcomes include: (1) rate of cellulitis-related hospital admissions; (2) percent change in leg volume from baseline, measured using the perometer; (3) QOL, assessed using the LYMQOL and EuroQol Five Dimension Scale (ED-5D-3L). The occurrence of cellulitis-related hospital admissions will be measured in the same manner as cellulitis recurrence.

Percent change in leg volume will be measured using a perometer, an optoelectronic imaging device designed to measure limb volume. ${ }^{27}$ The perometer has excellent intra-rater reliability (ICC $=1.0$; $95 \%$ CI: 0.99 to 1.00$)$ and inter-rater reliability ( $\mathrm{ICC}=1.0 ; 95 \% \mathrm{CI}: 0.97$ to 1.00$)$, is sensitive to changes in limb volume $e^{28-30}$ and is a valid measure of knee volume. ${ }^{27}$ Leg volume will be measured between 53 and $400 \mathrm{~mm}$ height from the ground using the perometer. Monthly calibration of the perometer will be conducted using a standardised object of known volume $(875 \mathrm{~mL})$ to minimise instrument error, ensuring consistency of this measurement device across the duration of the trial. Use of this device will also prevent potential differential measurement bias arising from lack of therapist blinding.

Where limb volume cannot be measured using the perometer, due to impaired mobility of a participant or equipment failure, summated circumferential leg measurements will be used following expert clinical guidelines. Circumferential leg measures will be taken at the mid foot, oblique ankle and at 10, 20, 30 and $40 \mathrm{~cm}$ intervals up the leg using a measurement board. Circumferential limb measurement also has excellent intra-rater reliability (ICC $=0.977-0.996 ; 95 \%$ CI: 0.960 to 0.998$)$ and inter-rater reliability (ICC $=0.942-0.994$; 95\% CI: 0.936 to 0.997$){ }^{31}$

QOL will be measured using LYMQOL, a validated, condition-specific QOL tool for people with lower limb lymphoedema, ${ }^{32}$ and the EQ-5D-3L, a generic preference-based measure of health-related QOL that comprises five dimensions of health. ${ }^{33}$ The EQ-5D can be used to calculate quality-adjusted life years for the purpose of economic evaluation. ${ }^{33}$ A systematic review has found the EQ-5D has good validity and responsiveness for people with skin diseases, although the tool has not been specifically validated within a population suffering cellulitis. ${ }^{33}$

Exploratory analysis will be conducted to test the robustness of the trial hypotheses and may include assessment of cellulitis recurrence post cross-over, intervention compliance, participant demographics, risk factors and per protocol analysis.

\section{Sample size and duration of follow-up}

The sample size has been calculated for the primary objective of detecting a difference in time to cellulitis recurrence between the control and intervention groups. The sample size estimation is based on the assumptions that the 3-year cellulitis recurrence rate in control participants is approximately $47 \%^{8}$ and compression therapy will reduce the 3-year incidence of recurrent cellulitis by $50 \%{ }^{17}{ }^{18}$ Assuming that events occur at a constant rate, these assumptions correspond to a hazard ratio (HR) of 0.42 . The eligibility criteria of two or more episodes of cellulitis in the same leg in the past 2 years has been used so that the trial cohort have an increased likelihood of cellulitis reoccurring during the follow-up period.

It is assumed that patients will be recruited over a 2.5 year period, and the total study duration will be 3.5 years. Length of participant follow-up will vary based on time of enrolment. Using a sequential design software package gsDesign in $\mathrm{R},{ }^{34}$ in order to detect a HR of 0.42 with $80 \%$ power and $2.5 \%$ (one-sided) type 1 error, a total of 45 cellulitis recurrences are needed. Under the present recruitment and recurrence assumptions, we plan to recruit 162 participants (81 per arm).

An interim analysis will be performed by a Data Monitoring Committee after 23 episodes of cellulitis. A log-rank test will be used to assess group differences. If a nominal (one sided) significance level of $\mathrm{p}=0.003$ is detected, indicating a strong clinical effect, the study will be ceased. If the Data Monitoring Committee recommends that the study continue to 45 episodes of cellulitis, 


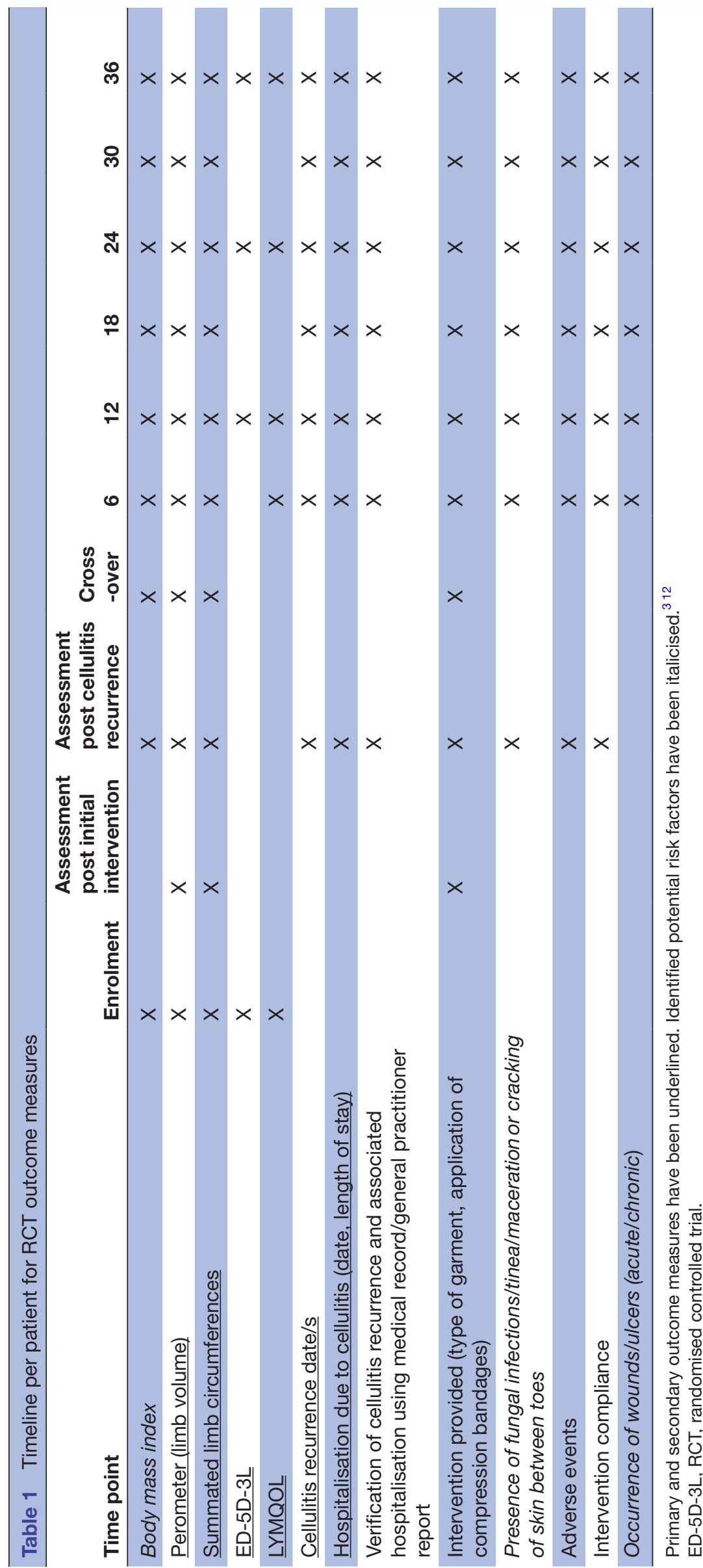


the final analysis will use a log-rank test with (one-sided) significance level $p=0.0238$. These efficacy bounds were derived using a Hwang-Shih-DeCani spending function with gamma $=-4$ to preserve an overall type 1 error rate of $5 \%$.

\section{Recruitment and enrolment of participants}

Recruitment will be conducted over a 2.5 -year period. A multifaceted recruitment strategy will be used. In order to capture acute patients (seen in CPHB and Canberra Hospital emergency departments and wards), all patients diagnosed with lower limb cellulitis during their hospital presentation will be sent information regarding the trial and how to contact the CPHB lymphoedema service if they would like to learn more information or self-refer. To recruit from the community, the study will be advertised via posters, radio and articles in various magazines and newspapers, providing information about the trial and encouraging self-referral. Education (in-services, faxes, newsletters and posters) and referral forms will be provided to recruitment sites (Canberra Hospital, CPHB, General Practices within the surrounding region) to encourage health professionals to refer patients. Patients from these sites must consent to a referral to the CPHB lymphoedema service for the study, but do not need to consent to participate in the trial at the time of referral.

After self-referral, a screening phone call will be conducted to check inclusion/exclusion criteria, and for those who appear to be eligible, an appointment at the service will be made with a lymphoedema therapist. At this appointment, candidates will be provided with participant information and consent forms, a verbal explanation of the study and an opportunity to ask questions, prior to choosing to consent or decline to participate.

To promote participation in the study, a free set of compression garments will be offered by a secondary sponsor. Compression garments are expensive, which can provide a barrier to treatment compliance. Participants in the intervention group will receive the free garments at intervention commencement. Participants in the control group will receive the free garments following their first cellulitis recurrence (cross-over) or on study completion for those who do not experience recurrence.

\section{Patient and public involvement}

A patient-centred approach was used to design this study. The trial design replicates the institution's standard clinical practice as closely as possible, while aiming to minimise any additional burden to participants. Patients from the participating clinical service were surveyed to assess acceptability of the model of care undertaken by the trial. As the time required to attend appointments was identified as a potential burden, the trial was designed to minimise scheduled follow-up appointments. Cost of compression therapy was identified as a likely financial burden which is minimised through the provision of two sets of free compression garments and use of accessible funding schemes. Referral processes were developed to enable patients to self-refer to the trial. The cross-over design feature was chosen to ensure participants do not continue to experience episodes of recurrent cellulitis without receiving the institution's standard intervention.

\section{Assignment of interventions and blinding}

Participants will be assigned to the intervention or control group in a 1:1 allocation ratio using block randomisation, with a block size of 10 . Sealed sequentially numbered opaque envelopes will be used to ensure concealed allocation. A computer-generated allocation sequence will be created and supplied by a consultant statistician and saved in a folder only accessible by administration staff. Administration staff will prepare the sealed sequentially numbered opaque envelopes, ensuring therapists involved in participant allocation have no premature access to the letters.

Therapists will not be blinded due to the practicalities of providing the intervention within a small team of four specialised clinicians. Further, the visible nature of the treatment and lack of feasible sham interventions prevent effective blinding of both assessors and participants. Additionally, for ethical reasons, participants will be fully informed of both the potential interventions, prior to consenting to participate.

\section{Data management and quality assurance}

Prior to any involvement in the trial, therapists will receive training regarding trial implementation and completion of outcome measures. Refresher training will be provided to therapists annually and the trial protocol will be kept readily available.

For the duration of the study, data will be stored in identifiable form in both a locked office and on a secure access hard drive, accessible only by designated research staff. Data will be entered by a research officer or members of the research team. For quality assurance, data completeness will be reviewed annually, and all entered data will be cross-checked against written records at least once after initial entry. Following trial conclusion and prior to data analysis, all data will be de-identified. Data will be stored for a minimum of 7 years as per CPHB policy, however data may be retained for longer for identified new, ethically approved ancillary studies. A contract with the secondary sponsor ensures that they will have no involvement in the study design, in the collection, analysis, or interpretation of data, in the writing of the manuscript or in the decision to submit the manuscript for publication.

\section{Participant retention}

Once a participant is enrolled in the study, every effort will be made to ensure they are followed up as per the protocol. Where participants cannot attend a scheduled appointment, a phone call assessment may be completed to gain the primary outcome measure. Phone call assessment will not allow for completion of limb volume or QOL measures but will capture the date of cellulitis recurrence and cellulitis-related hospitalisation. 
Participants can withdraw from the study at any point. For participants who withdraw, the medical record and/ or general practitioner report may be checked according to the schedule for cellulitis recurrence and cellulitis-related hospitalisation.

\section{Termination criteria}

Participants will be withdrawn from the study in the case of death, withdrawal of consent or if they develop a wound or lymphorrhoea requiring compression for effective management. ${ }^{35}$

\section{Proposed methods for data analysis}

For the main outcome measure of 'time to the first episode of recurrent cellulitis', survival analysis will be undertaken. Kaplan-Meier plots will be used to visualise patterns of time to first cellulitis recurrence between the groups, with a log-rank test being used to determine if there is a statistically significant difference between the groups. Cox proportional hazards regression may also be used to adjust for important risk factors. Right censoring will be used for participants who are lost to follow-up. Intention to treat analysis will be used, with all enrolled participants being assessed according to their randomisation, regardless of protocol adherence.

For the secondary outcomes of percent change in limb volume and QOL, measures will be taken at multiple time points. Therefore, groups will be compared using a linearmixed model or using a repeated measures analysis. A generalised linear model will be used to assess the rate of cellulitis-related hospital admissions.

\section{MINIMISING BIAS}

\section{Selection and attrition bias}

Use of randomisation will minimise selection bias and confounding. Stratification will ensure that the use of prophylactic antibiotics is not confounded with treatment assignment. The presence and distribution of other known potential confounding factors will be measured and reported. Intention to treat analysis will be used to prevent attrition bias that may occur through loss to follow-up of participants.

\section{Internal validity}

Use of an RCT and validated measurement tools support the internal validity of this research. The lack of blinding of therapists and participants has the potential to induce surveillance and recall bias and lead to differential measurement error in the reporting of cellulitis recurrence. To minimise this, the accuracy of self-report of recurrence may be cross-checked with the participant's general practitioner or medical record (from CPHB and Canberra Hospital). Diagnosis of cellulitis by doctors external to the study and use of perometry to measure limb volume will reduce the risk of measurement bias and thus differential measurement error. Calibration of the perometer will be performed to prevent non-differential measurement error that could result from machine error.

Control and intervention group participants have the same appointment schedule throughout the duration of the trial, however participants in the intervention group may attend more appointments than the control group. This systematic difference in clinician contact could influence the participant's perceived benefit, allowing potential bias in self-reported measures (LYMQOL, EQ-5D).

Participants enrolled in the trial have a history of two or more episodes of cellulitis diagnosed by medical practitioners independent to the trial. As misdiagnosis of lower limb cellulitis is not uncommon, ${ }^{36}$ the trial may include incorrectly diagnosed participants leading to non-differential misclassification.

\section{ANALYSIS OF COSTS}

A within-trial cost-analysis assessment will be conducted. Data obtained from the trial and participant medical records will be used to assess the cost of oedema management and the cost of an episode of cellulitis from both an individual and a health systems perspective. On completion of the RCT, the cost-effectiveness and cost-utility of chronic oedema management to prevent recurrent cellulitis may be assessed.

\section{ETHICS AND DISSEMINATION}

Ethics approval has been granted for these studies by three institutional committees:

1. Calvary Public Hospital Bruce Human Research Ethics Committee (53-2016).

2. Australian Capital Territory Health Human Research Ethics Committee (ETH.4.17.092).

3. University of Canberra Human Research Ethics Committee (cross-institutional approval).

Regardless of the outcome of the trial, the findings are planned to be submitted for publication in relevant peer-reviewed journals and for presentations at national and international conferences. Key findings will be disseminated to identified stakeholders, including primary contact clinicians for patients experiencing cellulitis (doctors and health professionals in acute and community settings), clinicians who manage chronic oedema and professionals who may be involved in developing relevant policy and practice. On request, participants will be provided with a copy of the trial results.

\section{DISCUSSION}

Although current expert consensus recommends compression therapy to prevent the recurrence of cellulitis in patients with lower limb chronic oedema, the evidence supporting this recommendation is lacking. This study aims to review the efficacy of compression therapy to allow for better-informed practice and policy. Given the high incidence of cellulitis within Australia and around the world, reducing cellulitis recurrence will significantly 
decrease the cost to the healthcare system and reduce the financial and personal burden of sufferers. Further, should compression therapy reduce the recurrence of cellulitis, this may limit the dependence and widespread prescription of prophylactic antibiotics. This trial will be performed on adults receiving healthcare services in the Australian Capital Territory, however, the results will be relevant to cellulitis management throughout Australia and internationally.

\section{Author affiliations}

${ }^{1}$ Department of Physiotherapy, Calvary Public Hospital Bruce, Canberra, Australian Capital Territory, Australia

${ }^{2}$ Discipline of Physiotherapy, Faculty of Health, University of Canberra, Canberra, Australian Capital Territory, Australia

${ }^{3}$ Biological Data Science Institute, Australian National University, Canberra, Australian Capital Territory, Australia

${ }^{4}$ University of Canberra Research Institute for Sport and Exercise (UC-RISE), University of Canberra, Canberra, Australian Capital Territory, Australia ${ }^{5}$ Calvary Public Hospital Bruce, Canberra, Australian Capital Territory, Australia ${ }^{6}$ Australian National University Medical School, Australian National University, Canberra, Australian Capital Territory, Australia

${ }^{7}$ Australian Institute of Health Innovation, Macquarie University, Sydney, New South Wales, Australia

Acknowledgements The authors acknowledge the Calvary Public Hospital Bruce Lymphoedema Team, specifically Gemma Arnold, Marie-Michelle Coulombe, Ingrid Thé, Abby Benton, Emma May, Sarah Squires, Caitlin Norris, Nievelle Chand, Sarah Toohey, Ashlee Cashion and Bhavleen Singh.

Contributors EW: trial design and implementation, contribution of original material, editing and approval of final manuscript. BB: trial implementation support and contribution of original material. TN: trial design input and statistical support. VM: economic analysis support. BB, TN, JG, FB and VM: provided supervision, contributed to refinement of the protocol and approved the final manuscript.

Funding Calvary Public Hospital Bruce is the primary sponsor, funding clinician time to initiate and manage the trial. Haddenham Healthcare is a secondary sponsor, providing two sets of free compression garments for each trial participant. Haddenham Healthcare had no role in designing this study and will not be involved in the trial implementation, analyses, data interpretation, or publication or dissemination of results. Haddenham Healthcare will not have access to trial data.

Competing interests None declared.

Patient consent for publication Not required.

Ethics approval The Human Research Ethics Committees of Calvary Public Hospital Bruce, Australian Capital Territory Health and University of Canberra all approved this trial.

Provenance and peer review Not commissioned; externally peer reviewed.

Open access This is an open access article distributed in accordance with the Creative Commons Attribution Non Commercial (CC BY-NC 4.0) license, which permits others to distribute, remix, adapt, build upon this work non-commercially, and license their derivative works on different terms, provided the original work is properly cited, appropriate credit is given, any changes made indicated, and the use is non-commercial. See: http://creativecommons.org/licenses/by-nc/4.0/.

\section{REFERENCES}

1. Swartz MN. Clinical practice. Cellulitis. N Engl J Med 2004;350:904-12.

2. Eriksson B, Jorup-Rönström C, Karkkonen K, et al. Erysipelas: clinical and bacteriologic spectrum and serological aspects. Clin Infect Dis 1996;23:1091-8.

3. Pavlotsky F, Amrani S, Trau H. Recurrent erysipelas: risk factors. J Dtsch Dermatol Ges 2004;2:89-95.

4. Inghammar M, Rasmussen M, Linder A. Recurrent erysipelas--risk factors and clinical presentation. BMC Infect Dis 2014;14:270-70.

5. The Australian Commission on Safety and Quality in Healthcare. Australian Atlas of Healthcare Variation 2017. 2017:81-90.
6. National Health Performance Authority. Healthy communities: potentially preventable hospitalisations in 2013-14. 2015

7. National Health Performance Authority. Hospital Performance: costs of acute admitted patients in public hospitals in 2011-12. 2015.

8. Cox NH. Oedema as a risk factor for multiple episodes of cellulitis/ erysipelas of the lower leg: a series with community follow-up. $\mathrm{Br} \mathrm{J}$ Dermatol 2006;155:947-50.

9. Jorup-Rönström C, Britton S. Recurrent erysipelas: predisposing factors and costs of prophylaxis. Infection 1987;15:105-6.

10. Mortimer PS, Levick JR. Chronic peripheral oedema: the critical role of the lymphatic system. Clin Med 2004;4:448-53.

11. Moffatt C, Keeley V, Quere I. The concept of chronic Edema-A neglected public health issue and an international response: the LIMPRINT study. Lymphat Res Biol 2019;17:121-6.

12. Dupuy A, Benchikhi $\mathrm{H}$, Roujeau JC, et al. Risk factors for erysipelas of the leg (cellulitis): case-control study. BMJ 1999;318:1591-4.

13. Karppelin M, Siljander T, Vuopio-Varkila J, et al. Factors predisposing to acute and recurrent bacterial non-necrotizing cellulitis in hospitalized patients: a prospective case-control study. Clin Microbiol Infect 2010;16:729-34.

14. Chlebicki MP, Oh CC, Cc O. Recurrent cellulitis: risk factors, etiology, pathogenesis and treatment. Curr Infect Dis Rep 2014;16:422.

15. Todd M. Chronic oedema: impact and management. Br J Nurs 2013;22:623-7.

16. Cox NH, Colver GB, Paterson WD. Management and morbidity of cellulitis of the leg. J R Soc Med 1998;91:634-7.

17. Arsenault K, Rielly L, Wise H. Effects of complete decongestive therapy on the incidence rate of hospitalization for the management of recurrent cellulitis in adults with lymphedema. Rehabil Oncol 2011;29:14-20.

18. Ko DS, Lerner R, Klose G, et al. Effective treatment of lymphedema of the extremities. Arch Surg 1998;133:452-8.

19. Thomas KS, Crook AM, Nunn AJ, et al. Penicillin to prevent recurrent leg cellulitis. N Engl J Med 2013;368:1695-703.

20. Dalal A, Eskin-Schwartz M, Mimouni D, et al. Interventions for the prevention of recurrent erysipelas and cellulitis. Cochrane Database Syst Rev 2017;6:CD009758.

21. Thomas K, Crook A, Foster K, et al. Prophylactic antibiotics for the prevention of cellulitis (erysipelas) of the leg: results of the UK Dermatology clinical trials network's PATCH II trial. Br J Dermatol 2012;166:169-78.

22. Sullivan T, de Barra E. Diagnosis and management of cellulitis. Clin Med 2018;18:160-3.

23. Raff AB, Kroshinsky D. Cellulitis: a review. JAMA 2016;316:325-37.

24. Australasian Lymphology Association. About the National Lymphoedema Practitioners Register. 2018 http://www. lymphoedema.org.au/the-register-updated (Accessed Sep 2018).

25. Carratalà J, Rosón B, Fernández-Sabé N, et al. Factors associated with complications and mortality in adult patients hospitalized for infectious cellulitis. Eur J Clin Microbiol Infect Dis 2003;22:151-7.

26. Nassaji M, Ghorbani R, Ghashghaee S. Risk factors of acute cellulitis in adult patients: a case-control study. EJM 2016;21:26-30.

27. Man IO, Markland KL, Morrissey MC. The validity and reliability of the perometer in evaluating human knee volume. Clin Physiol Funct Imaging 2004;24:352-8.

28. Czerniec SA, Ward LC, Lee MJ, et al. Segmental measurement of breast cancer-related arm lymphoedema using perometry and bioimpedance spectroscopy. Support Care Cancer 2011;19:703-10.

29. Czerniec SA, Ward LC, Refshauge KM, et al. Assessment of breast cancer-related arm lymphedema--comparison of physical measurement methods and self-report. Cancer Invest 2010;28:54-62

30. Bulley C, Coutts F, Tan CW. Perometry limb volume measurement: protocol development and reliability. Eur J Physiother 2013;15:193-200.

31. Devoogdt N, Lemkens H, Geraerts I, et al. A new device to measure upper limb circumferences: validity and reliability. Int Angiol 2010;29:401-7

32. Keeley $\mathrm{V}$, Crooks $\mathrm{S}$, Locke $\mathrm{J}$, et al. A quality of life measure for limb lymphoedema (LYMQOL). J lymphoedema 2010;5:26-37.

33. Yang $\mathrm{Y}$, Brazier J, Longworth L. EQ-5D in skin conditions: an assessment of validity and responsiveness. Eur J Health Econ 2015;16:927-39.

34. R Core Team. R: A language and environment for statistical computing: R Foundation for Statistical Computing, Vienna, Austria. 2018 https://www.R-project.org (Accessed Dec 2018).

35. O'Meara S, Cullum N, Nelson EA, et al. Compression for venous leg ulcers. Cochrane Database Syst Rev 2012;11:CD000265.

36. Weng QY, Raff AB, Cohen JM, et al. Costs and consequences associated with misdiagnosed lower extremity cellulitis. JAMA Dermatol 2017;153:141-6. 